\title{
Role of Vorticity in Generation of Pressure Sources and its Implication for Maintenance of Turbulence
}

\author{
B. Mikic
}

Received: 2 March 2012 / Accepted: 13 April 2012 / Published online: 1 May 2012

(C) The Author(s) 2012. This article is published with open access at SpringerLink.com

\begin{abstract}
The Poisson equation for pressure, together with the evolutions equations for the velocity gradients, reveals the role of vorticity in generation of pressure sources. Specifically, it was shown how a pressure field created by a local source, acting on nearby vorticity, would create new pressure sources. It was further established that a moving pressure field, which moves with the velocity of its source, but extends well beyond the source location, could lead to generation of fast and slow streaks as wells as contribute to formation of flow structures in the wall region. These processes, which are part of central mechanisms of maintenance of turbulence, suggest that turbulence could be self-sustaining only if the perturbation pressure force could overcome the diffusion effects; the value of friction Reynolds number reflects the balance between the two.
\end{abstract}

Keywords Turbulence • Vorticity and pressure sources • Coherent structures

\section{Key Properties of Poisson Equation for Pressure}

In the following, the symbols used are standard for the field, and the notation of Cartesian tensor assumes its common conventions. The coverage will be limited to wall turbulence, and specifically to flows between parallel plates of homogenous fluid without body forces. The mean flow is in the $\mathrm{x}$-direction, and the $\mathrm{y}$-coordinate is normal to the walls.

The Poisson equation for pressure shows that vorticity is an essential source for generation of pressure sources and it is the only source available for generation of negative sources.

B. Mikic $(\bowtie)$

Department of Mechanical Engineering, Massachusetts Institute of Technology,

Cambridge, MA 02139, USA

e-mail: boramikic@comcast.net 
Divergence of the momentum equation yields the Poisson equation for pressure,

$$
\nabla^{2} p=-S
$$

where the source term $\mathrm{S}$ is given as:

$$
S=\rho \frac{\partial u_{i}}{\partial x_{j}} \frac{\partial u_{j}}{\partial x_{i}}=\rho\left[\left(\frac{\partial u}{\partial x}\right)^{2}+\left(\frac{\partial v}{\partial y}\right)^{2}+\left(\frac{\partial w}{\partial z}\right)^{2}+2\left(\frac{\partial u}{\partial y} \frac{\partial v}{\partial x}+\frac{\partial u}{\partial z} \frac{\partial w}{\partial x}+\frac{\partial w}{\partial y} \frac{\partial v}{\partial z}\right)\right] .
$$

The following important features of the Poisson equations could be readily identified:

i) The length scale of a pressure field is always larger that the length scale of the source that created it;

ii) for $\mathrm{S}>0$, the source would increase nearby pressure, otherwise, for $\mathrm{S}<0$, it would lower the pressure;

iii) each of the three cross-product terms $\frac{\partial u_{i}}{\partial x_{j}} \frac{\partial u_{j}}{\partial x_{i}}$ with $i \neq j$, is comprised of two velocity gradients whose difference forms a vorticity component.

An obvious consequence of the last point is that in irrotational flows all the terms in the source tensor would be quadratic: if $\vec{\omega}=0$ then $\frac{\partial u_{i}}{\partial x_{j}}=\frac{\partial u_{j}}{\partial x_{i}}$ for any $i$ and $j$, and thus turbulent motions cannot exist in them.

Additionally, from the fact that if any of $\frac{\partial u_{i}}{\partial x_{j}} \frac{\partial u_{j}}{\partial x_{i}}$ terms were negative would imply a rollup of vorticity, follows a second corollary: the square root of the sum of squares of negative pressure source terms can be used to identify instantaneous locations of vortices.

\section{Evolution Equations for Velocity Gradients}

Each of the six source terms in the Poisson equation is comprised of products between two velocity gradients. This fact suggests that one could get a critical insight into generation of the sources by examining the evolution equations for the velocity gradients.

Applying the gradient operator to the momentum equation yields the evolution equations tensor for the gradients:

$$
\frac{D}{D t}\left(\frac{\partial u_{i}}{\partial x_{j}}\right)=-\frac{1}{\rho} \frac{\partial^{2} p}{\partial x_{i} \partial x_{j}}-\frac{\partial u_{k}}{\partial x_{j}} \frac{\partial u_{i}}{\partial x_{k}}+v \nabla^{2}\left(\frac{\partial u_{i}}{\partial x_{j}}\right) .
$$

There are two important inferences that follow from the above evolution equation. First, it is evident that second derivatives of pressure could create (or change) velocity gradients. Second, the mixed derivative of pressure $\frac{\partial^{2} p}{\partial x_{i} \partial x_{j}}, i \neq j$, would change the two gradients belonging to a single vorticity component, $\frac{\partial u_{i}}{\partial x_{j}}$ and $\frac{\partial u_{j}}{\partial x_{i}}$, for the same amount. As an example, we consider below the two gradients forming $\omega_{z}$; (analogous conclusions would hold for the other two vorticity components). Equation 2, 
together with the continuity equation, gives the respective evolution equations for the derivatives of $\frac{\partial v}{\partial x}$ and $\frac{\partial u}{\partial y}$ the following:

$$
\begin{aligned}
& \frac{D}{D t}\left(\frac{\partial v}{\partial x}\right)=-\frac{1}{\rho} \frac{\partial^{2} p}{\partial x \partial y}+\frac{\partial v}{\partial x} \frac{\partial w}{\partial z}-\frac{\partial v}{\partial z} \frac{\partial w}{\partial x}+v \nabla^{2}\left(\frac{\partial v}{\partial x}\right) \\
& \frac{D}{D t}\left(\frac{\partial u}{\partial y}\right)=-\frac{1}{\rho} \frac{\partial^{2} p}{\partial x \partial y}+\frac{\partial u}{\partial y} \frac{\partial w}{\partial z}-\frac{\partial u}{\partial z} \frac{\partial w}{\partial y}+v \nabla^{2}\left(\frac{\partial u}{\partial y}\right)
\end{aligned}
$$

The above relations show that, if in a material volume where originally only the $\frac{\partial u}{\partial y}$ component of $\omega_{z}$ were present, action of the mixed derivative of pressure $\frac{\partial^{2} p}{\partial x \partial y}$ would change the $\frac{\partial u}{\partial y}$ as well as create the $\frac{\partial v}{\partial x}$ gradient. This process of changing both gradients for the same amount would not change the value of the z-component of vorticity there, but significantly, it would create a new pressure source with its own contribution to the pressure field that would extend beyond the instantaneous location of the source, thus enabling generations of new sources. If $\frac{\partial^{2} p}{\partial x \partial y}<0$, the newly created source term would be positive; otherwise, for positive values of the mixed derivative of pressure, the source term would be negative.

Subtracting the evolution equation for $\frac{\partial u}{\partial y}$ from the one for $\frac{\partial v}{\partial x}$, after some rearrangement (including adding and subtracting the $\frac{\partial w}{\partial x} \frac{\partial w}{\partial y}$ term), returns the evolution equation for $\omega_{z}$ :

$$
\frac{D \omega_{z}}{D t}=\left(\omega_{x} \frac{\partial}{\partial x}+\omega_{y} \frac{\partial}{\partial y}+\omega_{z} \frac{\partial}{\partial z}\right) w+v \nabla^{2} \omega_{z}
$$

The above, of course, is identical with the expression that could be obtained directly from the evolution equation for the vorticity vector shown below.

$$
\frac{D \vec{\omega}}{D t}=\vec{\omega} \cdot \nabla \vec{V}+v \nabla^{2} \vec{\omega}
$$

The evolution equation for vorticity implies that vorticity could be generated solely on the fluid boundaries; (the first term on the right side simply states that the existing vorticity could be locally enhanced by stretching, or that each of the three vector components of vorticity could undergo tilting in other two directions).

The boundary fluxes of vorticity, which are the sole sources of vorticity, can be related to the pressure gradients on the wall trough the $\mathrm{x}$ and $\mathrm{z}$-direction momentum equations: Since on the solid boundary the following holds: $\left(\frac{\partial^{2} u}{\partial y^{2}}\right)_{0}=-\left(\frac{\partial \omega_{z}}{\partial y}\right)_{0}$, and $\left(\frac{\partial^{2} w}{\partial y^{2}}\right)_{0}=\left(\frac{\partial \omega_{x}}{\partial y}\right)_{0}$, the vorticity fluxes (into the fluid) follow from the respective momentum equations as:

$$
-v\left(\frac{\partial \omega_{z}}{\partial y}\right)_{0}=\frac{1}{\rho}\left(\frac{\partial p}{\partial x}\right)_{0}, \text { and, }-v\left(\frac{\partial \omega_{x}}{\partial y}\right)_{0}=-\frac{1}{\rho}\left(\frac{\partial p}{\partial z}\right)_{0}
$$

The vorticity that is generated would be comprised of a single velocity gradient term $\left(\frac{\partial u}{\partial y}\right.$ or $\left.\frac{\partial w}{\partial y}\right)$; only a subsequent action of the corresponding mixed derivative of pressure could create pressure sources, both positive and negative, depending on the sign of the derivatives. 
The source terms, ones formed, could be further augmented. It can be shown, using Eq. 2, that the cross products terms, with $i \neq j$, could be enhanced by stretching. For instance, using as an example $\frac{\partial u}{\partial y} \frac{\partial v}{\partial x}$ source term, from Eqs. 3a and 3b, the evolution equation for this source term, showing only (for the sake of brevity) the stretching component of the equation, has the following form: $\frac{D}{D t}\left(\frac{\partial u}{\partial y} \frac{\partial v}{\partial x}\right)=$ $2\left(\frac{\partial u}{\partial y} \frac{\partial v}{\partial x}\right) \frac{\partial w}{\partial z}+\ldots$

The above clearly indicates a potential for the exponential growth of the source strength due to stretching. This conclusion holds for both positive and negative values of the source term, that is, it is more general than vortex stretching effect that could be related only to the negative values of the source; each of the two gradients making a vorticity component could be individually enhanced by stretching even if they were not rolled-up into a vortex. This is clearly evident from the Eqs. $3 \mathrm{a}$ and $3 \mathrm{~b}$.

\section{On Generation of Flow Structures in the Wall Region}

Pressure sources are transportable quantities. The pressure fields they generate will move with the velocity of their source, but they will extend well beyond the instantaneous location of the source-they easily will reach the bounding walls. This means that intermittently there will be occurrences of a relative motion between a moving 3-D pressure field and the fluid in the wall regions; typically, a pressure field would be moving faster. One of the consequences of such an event will be development of fast and slow streaks in the wall regions as well as rotation of the surrounding fluid mass around the moving pressure field and the attendant formation of streamwise vortices.

The first effect follows directly from the evolution equation for the $\frac{\partial u}{\partial z}$, and the second would require also the evolution equation for streamwise vorticity.

The evolution equation for $\frac{\partial u}{\partial z}$ follows from relation 2 and the continuity equation as:

$$
\frac{D}{D t}\left(\frac{\partial u}{\partial z}\right)=-\frac{1}{\rho} \frac{\partial^{2} p}{\partial x \partial z}+\frac{\partial u}{\partial z} \frac{\partial v}{\partial y}-\frac{\partial u}{\partial y} \frac{\partial v}{\partial z}+v \nabla^{2}\left(\frac{\partial u}{\partial z}\right)
$$

Evidently, a presence of the mixed derivative of pressure $\frac{\partial^{2} p}{\partial x \partial z}$ in the wall region, which is imposed on the fluid by a moving 3-D pressure field, will generate the $\frac{\partial u}{\partial z}$ gradient that could lead to formation of fast and slow streak commensurate with the spanwise width of the moving pressure field. Furthermore, the action of the $\frac{\partial u}{\partial z}$ gradient would tilt $\omega_{z}$ in the x-direction, thus creating $\omega_{z}$. The latter effect is visible from the third term on the right side of the evolution equation for streamwise vorticity shown below.

$$
\frac{D \omega_{x}}{D t}=\left(\omega_{x} \frac{\partial}{\partial x}+\omega_{y} \frac{\partial}{\partial y}+\omega_{z} \frac{\partial}{\partial z}\right) u+v \nabla^{2} \omega_{x}
$$

The above sequential arguments support the following inference: a moving 3-D pressure field trough a wall region could lead to generation of fast and slow streaks there as well as contribute to rotation of a fluid mass around the $y$-axis, thus initiating conversion of the gradient $\frac{\partial u}{\partial y}$ of $\omega_{z}$ into $\pm \frac{\partial w}{\partial y}$ of $\omega_{x}$. 
Additional effects of a pressure field motion over the confining walls include generation of local pressure gradients around the edges of instantaneous 'footprints' of the moving pressure field. These gradients could reach very high intensities. For instance, in a DNS study at $\operatorname{Re}_{\tau}=180$, [1], it was found that the local maximum values of $\left(\frac{\partial p}{\partial x}\right)_{0}$ could be almost two orders of magnitude larger then the mean pressure gradient that drives the flow, $\frac{d \bar{p}}{d x}$; similar findings hold for the maximum values of $\left(\frac{\partial p}{\partial z}\right)_{0}$. The above findings indicate that in turbulent flows there would be a significant intermittent generation of local fluxes of vorticity on the boundaries. Thus, as follows from the relations 6 , the presence of local spanwise pressure gradients, $\left(\frac{\partial p}{\partial z}\right)_{0} \neq 0$, at two spanwise sides of a moving pressure field, implies flux of $\omega_{x}$ there. Obviously, the respective signs (orientations) of these vorticity will be different at each corresponding spanwise side of the pressure field, but each would have the same orientation as the local streamwise vorticity created by rotation of the fluid mass around the $\mathrm{y}$-axis that is induced by the $\frac{\partial u}{\partial z}$ gradient. Consequently, the two effects could act synchronously in formation of two counter-rotating strands of $\omega_{x}$ in the wall region.

Equally significant is the role of streamwise perturbation pressure gradients on the wall. Specifically, referring only to the lower wall (with an obvious modification analogous argument would hold for the upper wall), the presence of $\left(\frac{\partial p}{\partial x}\right)_{0}>0$ would create flux of $\omega_{z}$ that would have the counterclockwise orientation. Since the spanwise vorticity near the lower wall has the clockwise orientation, the generated vorticity would annihilate (by diffusion) some of the clockwise vorticity near the wall, thus creating a local maximum of $\omega_{z}$ (as well as the rate-of-strain maximum) away from the wall. The latter establishes conditions for a local rollup of displaced clockwise $\omega_{z}$ vorticity. Given that vorticity has solenoidal properties, this process, together with concurrent creation of two strands of $\omega_{x}$ by a moving pressure field, could play a role in formation of hairpin structures in the wall regions.

\section{Concluding Remarks}

Using for the first time the evolution equations for the velocity gradients, it was explicitly shown how a perturbation pressure field created by a local pressure source could generate fresh pressure sources, and more specifically, it revealed the role of vorticity in those processes. These findings, together with the processes involving relative motion between pressure fields and the fluid in the wall regions that could lead to formations of flow structures, point to contributing mechanisms for spreading and maintaining of turbulent motions.

To explore further the notion that processes of evolution of velocity gradients (and by extension, generation of pressure sources), are among the key features in maintenance of turbulent motions, one could compare competing terms in the evolution equations for velocity gradients, specifically, the pressure term (mixed derivative of pressure) and viscous term in Eq. 2. Using the established scaling in turbulent flows for perturbation pressure and velocities- the mean wall shear $\left(\bar{\tau}_{0}\right)$ for perturbation pressure, and the friction velocity $\left(\sqrt{\frac{\bar{t}_{0}}{\rho}}\right)$ for perturbation velocities- 
one gets that the pressure term scales with $\frac{\bar{\tau}_{0}}{\rho \Lambda^{2}}$, and the viscous term with $v \frac{\sqrt{\frac{\bar{t}_{0}}{\rho}}}{\Lambda^{3}} ; \Lambda$ above stands for the integral length scale of the system (the channel half-height for flows between parallel plates, the tube radius for flow in pipes, the boundary layer thickness for external flows).

Significantly, the ratio between the two yields the familiar friction Reynolds number, $\operatorname{Re}_{\tau}=\frac{\Lambda\left(\sqrt{\frac{\bar{t}_{0}}{\rho}}\right)}{v}$ which is already extensively used to denote intensity of turbulence in turbulent flows.

There is a strong indication that a $\mathrm{Re}_{\tau}$ value could be also an appropriate choice to demarcate a cutoff point below which turbulence could not be self-sustaining. Converting the accepted transition Re-numbers for various systems into $\mathrm{Re}_{\tau}$, using the laminar values for wall shear, consistently gives the corresponding values of $\operatorname{Re}_{\tau}$ at transition around 50. Moreover, experiments using modification of a basic flow by periodically transversely placing round cylinders in a parallel channel, a modification that will increase the amount of vorticity in the fluid as well as augment intensities of perturbation pressure events at a given flow rates, led to a significant reduction of Re-number (flow rate) at transition (up to fourfold), whereas, at the same time, the transition- $\mathrm{Re}_{\tau}$ values remained approximately invariant, [2, 3].

The above findings suggest that turbulent motions could be self-sustaining only when perturbation pressure forces could overcome the diffusion effects in generation of new pressure sources, that is, in changing or creating velocity gradients. Consistent with this inference is the fact that in the absence of internal pressure sources, laminar flows could persist well beyond the established values of transition Re-numbers.

Open Access This article is distributed under the terms of the Creative Commons Attribution License which permits any use, distribution, and reproduction in any medium, provided the original author(s) and the source are credited.

\section{References}

1. Kim, J.: On the structure of pressure fluctuations in simulated turbulent channel flow. J. Fluid Mech. 205, 421-451 (1989)

2. Mikic, B.B., Kapat, J.S., Ratnathicam, J.: Transition to turbulence in systems with eddy promoters: implication for the question of transition. Phys. Fluids A 4(9), 1854-1855 (1992)

3. Mikic, B.B., Vujisic, L.J.B., Kapat, J.S.: Turbulent transition and maintenance of turbulence; implication to heat transfer augmentation. Int. J. Heat Mass Tran. 37(Supl. 1), 425-431 (1994) 